\title{
ON SUMS OVER GAUSSIAN INTEGERS
}

\author{
BY
}

\author{
D. G. HAZLEWOOD
}

\begin{abstract}
The object of this paper is to give asymptotic estimates for some number theoretic sums over Gaussian integers. As a consequence of general estimates, asymptotic estimates with explicit error terms for the number of Gaussian integers with only "large" prime factors and for the number of Gaussian integers with only "small" prime factors are given.
\end{abstract}

1. Introduction. Let $G$ represent the set of Gaussian integers and let $P$ represent the set of Gaussian primes. Let $P_{1}$ denote the primes of $P$ that are in the interior of the first quadrant of the complex plane and let $P_{2}$ denote those primes of $P$ on the positive real axis. If $x \geqslant 1$ is a real number, let $C(x)$ denote the disc of radius $x$ (including the boundary) with center at the origin. Let $D(x)$ $=G \cap C(x), D_{1}(x)=P_{1} \cap C(x)$, and $D_{2}(x)=P_{2} \cap C(x)$. Let $k$ be a fixed natural number and fix $0=B_{0}<B_{1}<\cdots<B_{k-1}<B_{k}=+\infty$. A Gaussian integer $\alpha \in M_{n}, n=1, \ldots, k$, if either $\alpha=1$ or if all the Gaussian prime factors of $\alpha$ belong to

$$
\left(D_{1}\left(x^{B_{n}}\right) \cup D_{2}\left(x^{B_{n}}\right)\right) \cap\left(D_{1}\left(x^{B_{n-1}}\right) \cup D_{2}\left(x^{B_{n-1}}\right)\right)^{\sim}
$$

where $\sim$ is the complement operation with respect to the complex plane. Thus every $\alpha \in G$ can be uniquely expressed in the form

$$
\alpha=\mu \cdot \alpha_{1} \cdots \alpha_{k}
$$

where $\alpha_{n} \in M_{n}, n=1, \ldots, k$ and $\mu$ is a unit in $G$.

Let $f_{n}, n=1, \ldots, k$, denote completely multiplicative number theoretic functions. Then we define for a real number $t \geqslant 0$

$$
m_{f}\left(x^{t}\right)=\sum_{\alpha \in D\left(x^{t}\right)} f(N \alpha)=\sum_{\alpha \in D\left(x^{t}\right) ; \alpha=\mu \cdot \alpha_{1} \cdots \alpha_{k}} f_{1}\left(N \alpha_{1}\right) \cdots f_{k}\left(N \alpha_{k}\right)
$$

where $\mu$ is a unit, $\alpha_{n} \in M_{n}$, and $N \alpha=\alpha \cdot \bar{\alpha}=a^{2}+b^{2}$ if $\alpha=a+b i$ (the norm of $\alpha)$.

For example, if we define the function $\epsilon$ on the natural numbers by $\epsilon(n)=$

Received by the editors May 23, 1974.

AMS (MOS) subject classifications (1970). Primary 10-02, 10H15, $10 \mathrm{H} 25,10 \mathrm{H} 40$.

Key words and phrases. Gaussian integers, norms of Gaussian integers, numbers with small prime factors, numbers with large prime factors, differential-difference equations. 
1 if $n=1$ and $\epsilon(n)=0$ for $n>1$, then with $k=2, B_{1}=1, f_{1}(N \alpha)=\epsilon(N \alpha)$, and $f_{2}(N \alpha)=1$, we have

$$
m_{f}\left(x^{t}\right)=\Phi_{G}\left(x^{t}, x\right)
$$

where $\Phi_{G}\left(x^{t}, x\right)$ denotes the number of Gaussian integers in $C\left(x^{t}\right)$ with no Gaussian prime divisors in $C(x)$.

Similarly, if $k=2, B_{1}=1, f_{1}(N \alpha)=1$, and $f_{2}(N \alpha)=\epsilon(N \alpha)$, then

$$
m_{f}\left(x^{t}\right)=\Psi_{G}\left(x^{t}, x\right)
$$

where $\Psi_{G}\left(x^{t}, x\right)$ denotes the number of Gaussian integers in $C\left(x^{t}\right)$ with no Gaussian prime divisors outside $C(x)$.

As a consequence of some very general asymptotic estimates for (1.2), we prove asymptotic estimates for (1.3) and (1.4) that are uniform in $x$ and $t$ and exhibit an explicit error term. In particular, if $Z(t)$ is a function satisfying the differential-difference equation

$$
t Z^{\prime}(t)=a Z(t-1)
$$

with $a$ a real number and initial condition $Z(t)=1$ for $0 \leqslant t \leqslant 1(Z(t)=0$ for $t<0$ and $Z(t)$ is continuous at $t=1)$, then, as a special case of Theorem 2,

$$
\Phi_{G}\left(x^{t}, x\right)=2 \int_{2}^{2 t} x^{u} Z^{\prime}(u / 2) d u+O\left(x^{2 t} t^{C_{1}} \frac{H\left(x^{2}\right)}{\log x}\right)
$$

uniformly in $x$ and $t$, where $Z(t)$ satisfies (1.5) with $a=1, C_{1}$ is an absolute constant, and $H(x)$ is defined by (3.8). We should note that the constants implied by the use of the $O$-notation are absolute unless otherwise indicated.

As a special case of Theorem 5 , if $1 \leqslant t \leqslant(\log x)^{3 / 5-\delta}$ where $\delta$ is a positive real number, then

$$
\Psi_{G}\left(x^{t}, x\right)=\pi x^{2 t}\left\{Z(t)+o\left(t^{C^{4}} H\left(x^{2}\right)(\log x)^{C_{3}}+\frac{\left|Z^{\prime}(t-\epsilon)\right|}{\log x}\right)\right\}
$$

uniformly in $x$ and $t$ for $t$ outside the interval $(1,1+\epsilon)$, where $\epsilon$ is an arbitrary positive real number, $Z(t)$ satisfies (1.5) with $a=-1, C_{3}$ and $C_{4}$ are absolute constants, and $H(x)$ is defined by (3.8). We note that $Z(t)$ in (1.7) is the wellnown Dickman function.

Using (1.7) and the estimate by van der Corput in Landau [2] for the .umber of Gaussian integers in $C\left(x^{t}\right)$,

$$
\sum_{\alpha \in D\left(x^{t}\right)} 1=\pi x^{2 t}+o\left(x^{b t}\right)
$$

for $b<2 / 3$, we can give an estimate for the number of Gaussian integers in $C\left(x^{t}\right)$ 
with a prime divisor outside $C(x)$ as

$$
\pi x^{2 t}\left\{1-Z(t)+o\left(t^{C_{4}} H\left(x^{2}\right)(\log x)^{c_{3}}+\frac{\left|Z^{\prime}(t-\epsilon)\right|}{\log x}\right)\right\}
$$

for $1 \leqslant t \leqslant(\log x)^{3 / 5-\delta}, \delta>0$, which is proved by J. H. Jordan [1], but without the explicit error term.

2. Preliminary results. To estimate sums of the form (1.2), we follow the manner of B. V. Levin and A. S. Fainleib [3] and define for each function $f_{n}$, the function $\lambda_{f_{n}}$, by the following relation

$$
f_{n}(N \alpha) \log N \alpha=\sum_{\beta \mid \alpha}^{\prime} f_{n}(N \beta) \lambda_{f_{n}}\left(N \frac{\alpha}{\beta}\right)
$$

where the ' indicates that the sum is over only those Gaussian divisors of $\alpha$ that lie either in the interior of the first quadrant or on the positive real axis of the complex plane.

If $f$ is a convolution of functions $f_{1}$ and $f_{2}$, then $\lambda_{f}$ can be very simply expressed in terms of $\lambda_{f_{1}}$ and $\lambda_{f_{2}}$.

Lemma 1. Let

Then

$$
f(N \alpha)=\sum_{\beta \mid \alpha} f_{1}(N \beta) f_{2}\left(N \frac{\alpha}{\beta}\right)
$$

$$
\lambda_{f}(N \alpha)=\lambda_{f_{1}}(N \alpha)+\lambda_{f_{2}}(N \alpha) .
$$

Proof. Given $f, \lambda_{f}$ is uniquely determined by (2.1). In particular,

$$
4 \lambda_{f}(N \alpha)=\sum_{\beta \mid \alpha} f(N \beta) \tilde{f}\left(N \frac{\alpha}{\beta}\right) \log N \beta
$$

where $\widetilde{f}$ is defined by the relation

Now

$$
\sum_{\beta \mid \alpha}^{\prime} f(N \beta) \tilde{f}\left(N \frac{\alpha}{\beta}\right)=\epsilon(N \alpha) .
$$

$$
\begin{aligned}
\sum_{\beta \mid \alpha}^{\prime} f(N \beta) & \left(\lambda_{f_{1}}\left(N \frac{\alpha}{\beta}\right)+\lambda_{f_{2}}\left(N \frac{\alpha}{\beta}\right)\right) \\
= & \sum_{\beta \mid \alpha}^{\prime} \lambda_{f_{1}}\left(N \frac{\alpha}{\beta}\right) \sum_{\gamma \mid \beta} f_{1}\left(N \frac{\beta}{\gamma}\right) f_{2}(N \gamma)+\sum_{\beta \mid \alpha}^{\prime} \lambda_{f_{2}}\left(N \frac{\alpha}{\beta}\right) \sum_{\gamma \mid \beta} f_{1}(N \gamma) f_{2}\left(N \frac{\beta}{\gamma}\right) \\
= & \sum_{\gamma \mid \alpha} f_{2}(N \gamma) \sum_{\beta \mid \frac{\alpha}{\gamma}}^{\prime} f_{1}(N \beta) \lambda_{f_{1}}\left(N \frac{\alpha}{\beta \gamma}\right)+\sum_{\gamma \mid \alpha} f_{1}(N \gamma) \sum_{\beta \mid \frac{\alpha}{\gamma}}^{\prime} f_{2}(N \beta) \lambda_{f_{2}}\left(N \frac{\alpha}{\beta \gamma}\right) \\
= & \log N \alpha \sum_{\gamma \mid \alpha} f_{1}(N \gamma) f_{2}\left(N \frac{\alpha}{\gamma}\right)=\log N \alpha f(N \alpha) .
\end{aligned}
$$


Since $\lambda_{f}$ is uniquely determined, $\lambda_{f}(N \alpha)=\lambda_{f_{1}}(N \alpha)+\lambda_{f_{2}}(N \alpha)$ to prove Lemma 1 . Next we give a characterization of $\lambda_{f}$.

LEMMA 2. If $f$ is a completely multiplicative function, then

$$
\lambda_{f}(N \alpha)= \begin{cases}\log N \alpha f(N \alpha) & \text { if } \alpha=\rho^{r}, \rho \in P, \\ 0 & \text { if } \alpha \neq \rho^{r} .\end{cases}
$$

Proof. It is sufficient to show that $\lambda_{f}$ defined by (2.4) satisfies (2.1). Now

$$
\begin{aligned}
\sum_{\beta \mid \alpha}^{\prime} f\left(N \frac{\alpha}{\beta}\right) \lambda_{f}(N \beta) & =\sum_{\rho^{m}{ }_{\| \alpha}} \sum_{l=1}^{m} f\left(N \frac{\alpha}{\rho^{l}}\right) \lambda_{f}\left(N \rho^{l}\right) \\
& =\sum_{\rho^{m} \| \alpha}^{\prime} \sum_{l=1}^{m} f\left(N \frac{\alpha}{\rho^{l}}\right) \log N \rho f\left(N \rho^{l}\right) \\
& =f(N \alpha) \sum_{\rho^{m} \| \alpha}^{\prime} m \log N \rho=f(N \alpha) \log N \alpha .
\end{aligned}
$$

Again, since $\lambda_{f}$ is uniquely determined, we have proved the lemma.

We shall study the behavior of $m_{f}\left(x^{t}\right)$ for a class of functions $f$ determined by the conditional existence of the following two functions

$$
L_{f}(x, y)=4 \sum_{\rho^{r} \in D(x) ; \rho \in D(y)}^{\prime} \lambda_{f}\left(N \rho^{r}\right)=4 \sum_{\rho^{r} \in D(x) ; \rho \in D(y)}^{\prime} \log N \rho f\left(N \rho^{r}\right)
$$

(we note that $\rho^{r} \in D(x)$ implies $\rho \in D(x)$ and write $L_{f}(x, x)=L_{f}(x)$ ) and

$$
\Pi_{f}(x)=\prod_{\rho \in D(x)}^{\prime}\left(1+\sum_{r=1}^{\infty}\left|f\left(N \rho^{r}\right)\right|\right) .
$$

Another class of functions $f$ will be determined by conditions on

and

$$
\begin{aligned}
L_{f}^{*}(x, y) & =4 \sum_{\rho^{r} \in D(x) ; \rho \in D(y)}^{\prime} \lambda_{f}\left(N \rho^{r}\right) N \rho^{-r} \\
& =4 \sum_{\rho^{r} \in D(x) ; \rho \in D(y)}^{\prime} \log N \rho f\left(N \rho^{r}\right) N \rho^{-r}
\end{aligned}
$$

$$
\Pi_{f}^{*}(x)=\prod_{\rho \in D(x)}^{\prime}\left(1+\sum_{r=1}^{\infty}\left|f\left(N \rho^{r}\right)\right| N \rho^{-r}\right) .
$$

We now give the following fundamental requirements imposed on the functions $f_{n}, n=1, \ldots, k$ :

$$
L_{f_{n}}(x, y)=\tau_{n} \log \min (x, y)+D_{n}+h_{n}\left(x^{2}, y^{2}\right)
$$


where $\tau_{n}$ is a complex number, $D_{n}$ is an absolute constant, and $h_{n}(x, y)=$ $O(H(x)+H(y)), H(x)$ is a nonincreasing, nonnegative function; and

$$
\prod_{f_{n}}(x)=O\left(\log ^{A} n^{n}\right)
$$

where $A_{n}$ is an absolute constant.

We shall now prove the following lemma concerning (2.10) that will be needed later.

LEMMA 3. Let $f(N \alpha)=\Sigma_{\beta \mid \alpha} f_{1}(N \beta) f_{2}(N \alpha / \beta)$ where

$$
\Pi_{f_{1}}(x)=O\left(\log ^{A}{ }^{1} x\right) \text { and } \Pi_{f_{2}}(x)=O\left(\log ^{A} x\right) \text {, }
$$

then

$$
\Pi_{f}(x)=O\left(\log ^{A}{ }^{A}+A_{2} x\right) .
$$

Proof. Let $\rho \in P_{1} \cup P_{2}$, then

$$
\begin{aligned}
1+\sum_{r=1}^{\infty}\left|f\left(N \rho^{r}\right)\right| & \leqslant \sum_{r=0}^{\infty} \sum_{l=1}^{r}\left|f_{1}\left(N \rho^{l}\right)\right| \cdot\left|f_{2}\left(N \rho^{r-1}\right)\right| \\
& \leqslant\left(1+\sum_{r=1}^{\infty}\left|f_{1}\left(N \rho^{r}\right)\right|\right)\left(1+\sum_{r=1}^{\infty}\left|f_{2}\left(N \rho^{r}\right)\right|\right),
\end{aligned}
$$

so we conclude (2.11).

We are now ready to state and prove the basic result necessary to estimate $m_{f}\left(x^{t}\right)$.

Fundamental LEMma-Lemma 4. Suppose the completely multiplicative functions $f_{n}, n=1, \ldots, k$, satisfy (2.9). Then $m_{f}\left(x^{t}\right)$ as defined by (1.2) satisfies the equation

$$
\begin{aligned}
2 t m_{f}\left(x^{t}\right) & -2 \int_{0}^{t} m_{f}\left(x^{u}\right) d u \\
= & \frac{1}{4} \sum_{n=1}^{k} \tau_{n} \int_{t-B_{n}}^{t-B_{n-1}} m_{f}\left(x^{u}\right) d u
\end{aligned}
$$

$$
\begin{array}{r}
+\frac{D_{1}}{4 \log x} m_{f}\left(x^{t}\right)+\frac{1}{4 \log x} \sum_{\alpha \in D\left(x^{t}\right)} f(N \alpha) h_{1}\left(\frac{x^{2 t}}{N \alpha}, x^{2 B_{1}}\right) \\
+\frac{1}{4 \log x} \sum_{n=2}^{k} \sum_{\alpha \in D\left(x^{\left.t-B_{n-1}\right)}\right.} f(N \alpha)\left\{h_{n}\left(\frac{x^{2 t}}{N \alpha}, x^{2 B_{n}}\right)\right. \\
\left.-h_{n}\left(\frac{x^{2 t}}{N \alpha}, x^{2 B_{n-1}}\right)\right\} .
\end{array}
$$


Proof. Let

$$
\tilde{f}_{n}(N \alpha)= \begin{cases}f_{n}(N \alpha) & \text { if } \alpha \in M_{n}, \\ 0 & \text { if } \alpha \notin M_{n},\end{cases}
$$

then

$$
f(N \alpha)=\sum_{\alpha=\alpha_{1} \cdots \alpha_{k}} \tilde{f_{1}}\left(N \alpha_{1}\right) \cdots \widetilde{f_{k}}\left(N \alpha_{k}\right) .
$$

Therefore, using Lemma 1 and (2.9), we see that

$$
\begin{aligned}
L_{f}\left(x^{t}\right)= & 4 \sum_{\rho^{r} \in D\left(x^{t}\right)}^{\prime} \lambda_{f}\left(N \rho^{\eta}\right) \\
= & 4 \sum_{n=1}^{k} \sum_{\rho^{r} \in D\left(x^{t}\right)}^{\prime} \lambda_{\tilde{f}_{n}}\left(N \rho^{\eta}\right)=4 \sum_{n=1}^{k} \sum_{\rho^{r} \in D\left(x^{t}\right) ; \rho \in M_{n}} \lambda_{f_{n}}\left(N \rho^{\eta}\right) \\
= & \sum_{n=1}^{k} \tau_{n} \log \left(\frac{\min \left(x^{t}, x^{B_{n}}\right)}{\min \left(x^{t}, x^{B_{n-1}}\right)}\right)+D_{1}+h_{1}\left(x^{2 t}, x^{2 B_{1}}\right) \\
& +\sum_{n=2}^{k}\left\{h_{n}\left(x^{2 t}, x^{2 B_{n}}\right)-h_{n}\left(x^{2 t}, x^{2 B_{n-1}}\right)\right\} .
\end{aligned}
$$

Now using Theorem 1.4 of Prachar [4, p. 371], we have

$$
\begin{aligned}
\sum_{\alpha \in D\left(x^{t}\right)} f(N \alpha) \log N \alpha & =\sum_{N \alpha<x^{2 t}} f(N \alpha) \log N \alpha \\
& =m_{f}\left(x^{t}\right) \log x^{2 t}-\int_{1}^{x^{2 t}} m_{f}(\sqrt{v}) \frac{d v}{v} \\
& =m_{f}\left(x^{t}\right) \log x^{2 t}-2 \log x \int_{0}^{t} m_{f}\left(x^{u}\right) d u .
\end{aligned}
$$

Thus, using the definition of $\lambda_{f}$,

$$
\begin{aligned}
& m_{f}\left(x^{t}\right) \log x^{2 t}-2 \log x \int_{0}^{t} m_{f}\left(x^{u}\right) d u \\
& =\sum_{\alpha \in D\left(x^{t}\right)} \sum_{\beta \mid \alpha}^{\prime} f(N \beta) \lambda_{f}\left(N \frac{\alpha}{\beta}\right) \\
& =\frac{1}{4} \sum_{\alpha \in D\left(x^{t}\right)} \sum_{\beta \mid \alpha} f(N \beta) \lambda_{f}\left(N \frac{\alpha}{\beta}\right)=\frac{1}{4} \sum_{\beta \in D\left(x^{t}\right)} f(N \beta) L_{f}\left(\frac{x^{t}}{\sqrt{N \beta}}\right)
\end{aligned}
$$




$$
\begin{aligned}
& =\sum_{\alpha \in D\left(x^{t}\right)} f(N \alpha) \frac{1}{4}\left\{\sum_{n=1}^{k} \tau_{n} \log \left(\frac{\min \left(x^{t} / \sqrt{N \alpha}, x^{B_{n}}\right)}{\min \left(x^{t} / \sqrt{N \alpha}, x^{B_{n}-1}\right)}\right)+D_{1}+h_{1}\left(\frac{x^{2 t}}{N \alpha^{\prime}} x^{2 B_{1}}\right)\right. \\
& \left.\quad+\sum_{n=2}^{k}\left(h_{n}\left(\frac{x^{2 t}}{N \alpha}, x^{2 B_{n}}\right)-h_{n}\left(\frac{x^{2 t}}{N \alpha}, x^{2 B_{n}-1}\right)\right)\right\} \\
& =\frac{1}{4} \sum_{n=1}^{k} \tau_{n} \sum_{\alpha \in D\left(x^{t}\right)} f(N \alpha) \log \left(\frac{\min \left(x^{t} / \sqrt{N \alpha}, x^{B_{n}}\right)}{\min \left(x^{t} / \sqrt{N \alpha}, x^{B_{n}-1}\right)}\right) \\
& +\frac{D_{1}}{4} m_{f}\left(x^{t}\right)+\frac{1}{4} \sum_{\alpha \in D\left(x^{t}\right)} f(N \alpha) h_{1}\left(\frac{x^{2 t}}{N \alpha}, x^{2 B_{1}}\right) \\
& +\frac{1}{4} \sum_{n=2}^{k} \sum_{\alpha \in D\left(x^{t-B_{n-1}}\right)} f(N \alpha)\left\{h_{n}\left(\frac{x^{2 t}}{N \alpha}, x^{2 B_{n}}\right)-h_{n}\left(\frac{x^{2 t}}{N \alpha}, x^{2 B_{n-1}}\right)\right\} .
\end{aligned}
$$

Thus changing the first sum on the right-hand side to an integral and changing the variable, we derive (2.12).

We shall need two other lemmas in $\S 3$ that are proved in Levin and Fainleib [3].

LemMa 5 [3, LeMma 1.2.1]. Let $R(t, x)$ be a complex-valued function of real variables $t$ and $x$ integrable with respect to $t$; let $a$ and $b_{1}, \ldots, b_{m}$ be complex numbers, $C_{1} \geqslant 0$, and $0 \leqslant B_{0}<B_{1}<\cdots<B_{m}<+\infty$. Suppose further that $R(t, x)=0$ for $t \leqslant 0$ and that

$$
t R(t, x)-(a+1) \int_{0}^{t} R(u, x) d u+\sum_{n=1}^{m} b_{n} \int_{t-B_{n}}^{t-B_{n-1}} R(u, x) d u=O\left(t^{C_{1}}\right)
$$

uniformly in $x$. If $\int_{0}^{\lambda}|R(u, x)| d u=O(1)$ uniformly in $x$, where $\lambda$ is a positive constant, then there exists a constant $C_{2}>0$ such that for all $t \geqslant \lambda$

uniformly in $x$.

$$
R(t, x)=O\left(t^{C_{2}}\right)
$$

LemmA 6 (Argument on pp. 174-175 of [3]). Let $0<B_{1}<\cdots<B_{m}<$ $+\infty$ and $\tau_{1}, \ldots, \tau_{m}$ denote complex numbers. If $Z(t)$ satisfies the equation

$$
t Z^{\prime}(t)=\sum_{n=1}^{m-1}\left(\tau_{n+1}-\tau_{n}\right) Z\left(t-B_{n}\right)
$$

with initial condition $Z(t)=1$ for $0 \leqslant t \leqslant B_{1}$ and $\hat{Z}(t)$ satisfies the equation

$$
t \hat{Z}^{\prime}(t)=\sum_{n=1}^{m-1}\left(\tau_{n}-\tau_{n+1}\right) \hat{Z}\left(t-B_{n}\right)
$$


with initial condition $\hat{Z}(t)=1$ for $0 \leqslant t \leqslant B_{1}$, then

$$
\int_{0}^{t} Z^{\prime}(t-u) \hat{Z}^{\prime}(u) d u+Z^{\prime}(t)+\hat{Z}^{\prime}(t)=0 .
$$

Using the fundamental lemma, Lemma 4, we are now ready to derive asymptotic estimates for $m_{f}\left(x^{t}\right)$.

3. Estimates for $m_{f}\left(x^{t}\right)$. The first estimate for $m_{f}\left(x^{t}\right)$ is the special case where the sum is taken over those Gaussian integers with only "large" prime factors.

THEOREM 1. Let $f_{n}, n=1, \ldots, k$, be completely multiplicative functions satisfying (2.9) and (2.10) where $f_{1}(N \alpha)=\epsilon(N \alpha)$ and $A=\Sigma_{n=1}^{k} A_{n}$. Then

$$
m_{f}\left(x^{t}\right)=4 Z(t)+O\left(t^{A} k^{\cdot \Delta} H\left(x^{2 B_{1}}\right)(\log x)^{A-1}\right)
$$

uniformly in $x$ and $t$, where $Z(t)$ satisfies the equation

$$
t Z(t)-\int_{0}^{t} Z(u) d u-\sum_{n=2}^{k} \frac{\tau_{n}}{8} \int_{t-B_{n}}^{t-B_{n-1}} Z(u) d u=0
$$

with initial condition $Z(t)=1$ for $0 \leqslant t \leqslant B_{1}$, and

$$
\Delta=\left\{\begin{array}{l}
1 \text { if } f_{k}(N \alpha) \neq \epsilon(N \alpha), \\
0 \text { if } f_{k}(N \alpha)=\epsilon(N \alpha) .
\end{array}\right.
$$

Proof. Let $\tau_{1}=D_{1}=0, h_{1}\left(x^{2}, y^{2}\right)=0$. Therefore (2.12) can be written as

$$
\begin{aligned}
2 t m_{f}\left(x^{t}\right)-2 \int_{0}^{t} m_{f}\left(x^{u}\right) d u-\sum_{n=2}^{k} \frac{\tau_{n}}{4} \int_{t-B_{n}}^{t-B_{n-1}} m_{f}\left(x^{u}\right) d u \\
=\frac{1}{4 \log x} \sum_{n=2}^{k} \sum_{\alpha \in D\left(x^{t-B_{n-1}}\right)} f(N \alpha)\left\{h_{n}\left(\frac{x^{2 t}}{N \alpha}, x^{2 B_{1}}\right)-h_{n}\left(\frac{x^{2 t}}{N \alpha}, x^{2 B_{n-1}}\right)\right\} .
\end{aligned}
$$

Now $\Pi_{f_{n}}(x)=O\left(\log ^{A} n_{x}\right)$ for $n=2, \ldots, k$ so that

Hence

$$
m_{f}\left(x^{t}\right) \leqslant \sum_{\alpha \in D\left(x^{t}\right)}|f(N \alpha)|=O\left(t^{A} k^{\cdot \Delta} \log { }^{A} x\right) .
$$

$$
\begin{aligned}
2 t m_{f}\left(x^{t}\right)-2 \int_{0}^{t} m_{f}\left(x^{u}\right) d u-\sum_{n=2}^{k} \frac{\tau_{n}}{4} \int_{t-B_{n}}^{t-B_{n-1}} m_{f}\left(x^{u}\right) d u \\
=O\left(t^{A} k^{\cdot \Delta}(\log x)^{A-1} H\left(x^{2 B_{1}}\right)\right) .
\end{aligned}
$$


We let

$$
m_{f}\left(x^{t}\right)=4 Z(t)+R(t, x) H\left(x^{2 B_{1}}\right)(\log x)^{A-1}
$$

so that

$$
\begin{aligned}
& 2 t m_{f}\left(x^{t}\right)-2 \int_{0}^{t} m_{f}\left(x^{u}\right) d u-\sum_{n=2}^{k} \frac{\tau_{n}}{4} \int_{t-B_{n}}^{t-B_{n-1}} m_{f}\left(x^{u}\right) d u \\
& =8 t Z(t)-8 \int_{0}^{t} Z(u) d u-\sum_{n=2}^{k} \tau_{n} \int_{t-B_{n}}^{t-B_{n-1}} Z(u) d u \\
& +H\left(x^{2 B_{1}}\right)(\log x)^{A-1}\left\{2 t R(t, x)-2 \int_{0}^{t} R(u, x) d u\right. \\
& \left.\quad-\sum_{n=2}^{k} \frac{\tau_{n}}{4} \int_{t-B_{n}}^{t-B_{n-1}} R(u, x) d u\right\} .
\end{aligned}
$$

Using Lemma 5 with

$$
t R(t, x)-\int_{0}^{t} R(u, x) d u-\sum_{n=2}^{k} \frac{\tau_{n}}{8} \int_{t-B_{n}}^{t-B_{n-1}} R(u, x) d u=O\left(t^{A}{ }^{*} \cdot \Delta\right)
$$

uniformly in $x$ and with $\lambda=B_{1}, \int_{0}^{B_{1}} R(u, x) d u=O(1)$ uniformly in $x$, then there exists an absolute constant $C_{2}$ such that $R(t, x)=O\left(t^{C_{2}}\right)$ uniformly in $x$. Hence we prove Theorem 1.

We note that condition (2.10) is needed only if the $f_{n}$ can be negative; hence for nonnegative $f_{n}$ we have the following

COROLlaRY. If the $f_{n}$ are nonnegative, satisfying (2.9) and $f_{1}(N \alpha)=$ $\epsilon(N \alpha)$, then

$$
m_{f}\left(x^{t}\right)=4 Z(t)+o\left(t^{\operatorname{Re} \tau_{k} \cdot \Delta} \frac{H\left(x^{2 B_{1}}\right)}{\log x}\right)
$$

uniformly in $x$ and $t$.

Using Abel's summation on (3.1), we obtain the following theorem which gives the asymptotic estimate for $\Phi_{G}\left(x^{t}, x\right)$.

THEOREM 2. Let $f_{1}(N \alpha)=\epsilon(N \alpha)$ and $f_{n}, n=2, \ldots, k$, be completely multiplicative functions satisfying

$$
L_{f_{n}}^{*}(x, y)=\tau_{n} \log \min (x, y)+D_{n}+h_{n}\left(x^{2}, y^{2}\right)
$$

and

$$
\Pi_{f_{n}}^{*}(x)=O\left(\log ^{A}{ }^{n} x\right)
$$


Then

$$
m_{f}\left(x^{t}\right)=2 \int_{2 B_{1}}^{2 t} x^{u} Z^{\prime}\left(\frac{u}{2}\right) d u+O\left(x^{2 t} t^{A} k^{\cdot \Delta} H\left(x^{2 B_{1}}\right)(\log x)^{A-1}\right)
$$

uniformly in $x$ and $t$, where $A, A_{k}, Z(t)$, and $\Delta$ are defined in Theorem 1.

For the estimate for $\Phi_{G}\left(x^{t}, x\right)$ given in (1.6), we make the following considerations in Theorem 2:

Let $k=2, B_{1}=1, f_{1}(N \alpha)=\epsilon(N \alpha), f_{2}(N \alpha)=1$, then

$$
L_{f_{2}}^{*}(x, y)=8 \log \min (x, y)+D+O\left(H\left(x^{2}\right)+H\left(y^{2}\right)\right)
$$

where

$$
H(x)=O\left(\exp \left(-C(\log x)^{3 / 5-\delta}\right)\right)
$$

with $C>0, \delta>0$ fixed, by the following argument. Now

$$
\begin{aligned}
\frac{1}{4} L_{f_{2}}^{*}(x, y) & =\sum_{\rho^{r} \in D(x) ; \rho \in D(y)}^{\prime} \frac{\log N \rho}{N \rho^{r}} \\
= & \sum_{N \rho^{r}<x^{2} ; N \rho \leqslant y^{2} ; \rho \in P_{1}} \frac{\log N \rho}{N \rho^{r}} \lambda+\sum_{N \rho^{r} \leqslant x^{2} ; N \rho \leqslant y^{2} ; \rho \in P_{2}} \frac{\log N \rho}{N \rho^{r}} \\
= & \sum_{1}+\sum_{2} .
\end{aligned}
$$

If $\rho \in P_{2}$, then $\rho=p$ is a rational prime with $p \equiv 3(\bmod 4)$ and $N \rho=p^{2}$. If $\rho \in P_{1}, \rho \neq 1+i$, then there exists a rational prime $p, p \equiv 1(\bmod 4)$, and $N \rho=$ $p$; and conversely, for each rational prime $p, p \equiv 1(\bmod 4)$, there exist exactly two primes $\rho_{1}$ and $\rho_{2}$ in $P_{1}$ such that $p=N \rho_{1}=N \rho_{2}$. Using this information, we see that

$$
\sum_{2}=2 \underset{p^{r} \leqslant x ; p \leqslant y ; p \equiv 3(\bmod 4)}{ } \frac{\log p}{p^{2}} \leqslant C
$$

where $C$ is an absolute constant, and

$$
\begin{aligned}
\sum_{1} & =D^{\prime}+\sum_{p^{r}<x^{2} ; p<y^{2} ; p \equiv 1(\bmod 4)} \frac{\log p}{p^{r}} \\
& =2 \log \min (x, y)+D^{\prime \prime}+O\left(H\left(x^{2}\right)+H\left(y^{2}\right)\right)
\end{aligned}
$$

where $D^{\prime \prime}$ is an absolute constant and $H(x)$ is defined by (3.8). Hence $\tau_{2}=8$, and using this in (3.6), we get (1.6).

Now we shall give a formula for the general case of $m_{f}\left(x^{t}\right)$. 
THEOREM 3. Let $f_{n}, n=1, \ldots, k$, be completely multiplicative functions satisfying (2.9) and (2.10). Then

$$
m_{f}\left(x^{t}\right)=m_{f_{1}}\left(x^{t}\right)+\int_{0}^{t} Z^{\prime}(t-u) m_{f_{1}}\left(x^{u}\right) d u+O\left(t^{C_{4}} H\left(x^{2 B_{1}}\right)(\log x)^{C_{3}}\right)
$$

uniformly in $x$ and $t$, where

$$
m_{f_{1}}\left(x^{t}\right)=\sum_{\alpha \in D\left(x^{t}\right)} f_{1}(N \alpha),
$$

$Z(t)$ satisfies the differential-difference equation

$$
t Z^{\prime}(t)=\frac{1}{8} \sum_{n=1}^{k-1}\left(\tau_{n+1}-\tau_{n}\right) Z\left(t-B_{n}\right)
$$

with initial condition $Z(t)=1$ for $0 \leqslant t \leqslant B_{1}$, and $C_{3}$ and $C_{4}$ are absolute constants.

Proof. We define functions $\hat{f}_{n}, n=1, \ldots, k$, by the relations

$$
\sum_{\beta \mid \alpha}^{\prime} \hat{f}_{n}(N \beta) f_{n}\left(N \frac{\alpha}{\beta}\right)=f_{1}(N \alpha) .
$$

Hence for $n=1$, (3.13) implies that

$$
\hat{f}_{1}(N \alpha)=\epsilon(N \alpha) \text {. }
$$

Further, using Lemma 1 and (2.9),

$$
\begin{aligned}
L_{f_{n} / 4}(x, y) & =L_{f_{1}}(x, y)-L_{f_{n}}(x, y) \\
& =\left(\tau_{1}-\tau_{n}\right) \log \min (x, y)+D_{1}-D_{n}+O\left(H\left(x^{2}\right)+H\left(y^{2}\right)\right) .
\end{aligned}
$$

By Lemma 3,

$$
\Pi_{\hat{f}_{n} / 4}(x)=O\left(\log ^{\dot{A}} n x\right)
$$

Thus, the conditions of Theorem 1 are satisfied using the functions $\hat{f}_{1}, 1 / 4 \hat{f}_{2}, \ldots$, $1 / 4 \hat{f}_{k}$. Therefore

$$
\text { 4 } \begin{aligned}
4^{-k} \cdot m_{\hat{f}}\left(x^{t}\right) & =\frac{1}{4} \sum_{\alpha \in D\left(x^{t}\right) ; \alpha=\mu \alpha_{1} \cdots \alpha_{k}} \hat{f}_{1}\left(N \alpha_{1}\right) \cdot \frac{1}{4} \hat{f}_{2}\left(N \alpha_{2}\right) \cdots \frac{1}{4} \hat{f}_{k}\left(N \alpha_{k}\right) \\
& =\hat{Z}(t)+O\left(t^{A}{ }^{A} \cdot{ }^{2} H\left(x^{2 B_{1}}\right)(\log x)^{A-1}\right)
\end{aligned}
$$

where $\hat{Z}(t)$ satisfies the equation

$$
t \hat{Z}^{\prime}(t)=\frac{1}{8} \sum_{n=1}^{k-1}\left(\tau_{n}-\tau_{n+1}\right) \hat{Z}\left(t-B_{n}\right)
$$

with initial condition $\hat{Z}(t)=1$ for $0 \leqslant t \leqslant B_{1}$.

Now we consider the following sum 


$$
\begin{aligned}
& m_{f_{1}}\left(x^{t}\right)=\sum_{\alpha \in D\left(x^{t}\right)} f_{1}(N \alpha)=\sum_{\alpha \in D\left(x^{t}\right) ; \alpha=\mu \cdot \alpha_{1} \cdots \alpha_{k}} f_{1}\left(N \alpha_{1}\right) \cdots f_{1}\left(N \alpha_{k}\right) \\
& =\sum_{\substack{\alpha \in D\left(x^{t}\right), \alpha=\mu \cdot \alpha_{1} \cdots \alpha_{k}}} \sum_{\beta_{1} \mid \alpha_{1}}^{\prime} f_{1}\left(N \beta_{1}\right) \hat{f}_{1}\left(N \frac{\alpha_{1}}{\beta_{1}}\right) \cdots \sum_{\beta_{k} \mid \alpha_{k}}^{\prime} f_{k}\left(N \beta_{k}\right) \hat{f}_{k}\left(N \frac{\alpha_{k}}{\beta_{k}}\right) \\
& =4^{-k} \sum_{\substack{\beta \in D\left(x^{t}\right) \\
\beta=\mu \cdot \beta_{1} \cdots \beta_{k}}} f_{1}\left(N \beta_{1}\right) \cdots f_{k}\left(N \beta_{k}\right) \sum_{\substack{\alpha \in D(x) \\
\alpha=\mu \cdot \alpha_{1} \cdots \alpha_{k} \\
\beta_{i} \mid \alpha_{i}, 1<i \leqslant k}} f_{1}\left(N \frac{\alpha_{1}}{\beta_{1}}\right) \cdots \hat{f}_{k}\left(N \frac{\alpha_{k}}{\beta_{k}}\right) \\
& =4^{-k} \sum_{\substack{\beta \in D\left(x^{t}\right) \\
\beta=\mu \cdot \beta_{1} \cdots \beta_{k}}} f_{1}\left(N \beta_{1}\right) \cdots f_{k}\left(N \beta_{k}\right) \sum_{\substack{\left.\gamma \in D\left(x^{t}\right) / \sqrt{N \beta}\right) \\
\gamma=\mu \cdot \gamma_{1} \cdots \gamma_{k}}} \hat{f}_{1}\left(N \gamma_{1}\right) \cdots \hat{f}_{k}\left(N \gamma_{k}\right) \\
& =4^{-k} \sum_{\substack{\beta \in D\left(x^{t}\right) \\
\beta=\mu \cdot \beta_{1} \cdots \beta_{k}}} f_{1}\left(N \beta_{1}\right) \cdots f_{k}\left(N \beta_{k}\right) m_{\hat{f}}\left(\frac{x^{t}}{\sqrt{N \beta}}\right) .
\end{aligned}
$$

Hence, using (3.17),

$$
\begin{aligned}
& m_{f_{1}}\left(x^{t}\right)=\sum_{\alpha \in D\left(x^{t}\right)} f_{1}\left(N \alpha_{1}\right) \cdots f_{k}\left(N \alpha_{k}\right) \hat{z}\left(t-\frac{\log N \alpha}{2 \log x}\right) \\
& \alpha=\alpha_{\alpha \cdot \alpha_{1}}^{\left.\alpha \in x^{t}\right)} \alpha_{k} \\
& +O\left(t^{A 1^{-\Delta}} H\left(x^{2 B_{1}}\right)(\log x)^{A-1} \sum_{\substack{\alpha \in D\left(x^{t}\right) \\
\alpha=\mu \cdot \alpha_{1} \cdots \alpha_{k}}}\left|f_{1}\left(N \alpha_{1}\right) \cdots f_{k}\left(N \alpha_{k}\right)\right|\right) \\
& =m_{f}\left(x^{t}\right)+\sum \quad f_{1}\left(N \alpha_{1}\right) \cdots f_{k}\left(N \alpha_{k}\right) \int_{0}^{t-(\log N \alpha) / 2 \log x} \hat{Z}^{\prime}(u) d u \\
& \alpha \stackrel{\alpha \in D\left(\alpha_{1} \cdots \alpha_{k}\right.}{\omega_{1}} \\
& +O\left(t^{C_{2}} H\left(x^{2 B_{1}}\right)(\log x)^{C_{3}}\right),
\end{aligned}
$$

which is equivalent to

$$
\begin{aligned}
m_{f_{1}}\left(x^{t}\right)= & m_{f}\left(x^{t}\right)+\int_{0}^{t} \dot{Z}^{\prime}(t-u) m_{f}\left(x^{u}\right) d u \\
& +O\left(t^{C_{2}} H\left(x^{2 B_{1}}\right)(\log x)^{C_{3}}\right) .
\end{aligned}
$$

From Lemma 6 we see that

$$
\int_{0}^{t} Z^{\prime}(t-u) \hat{Z}^{\prime}(u) d u+Z^{\prime}(t)+\hat{Z}^{\prime}(t)=0 .
$$

Using (3.19) and (3.20), it follows that 


$$
\begin{aligned}
\int_{0}^{t} Z^{\prime}(t-u) m_{f_{1}}\left(x^{u}\right) d u \\
=\int_{0}^{t} Z^{\prime}(t-u) m_{f}\left(x^{u}\right)+\int_{0}^{t} m_{f}\left(x^{u}\right) \int_{0}^{t-u} Z^{\prime}(t-u-v) \hat{Z}^{\prime}(v) d v d u \\
\quad+O\left(t^{C_{4}} H\left(x^{2 B_{1}}\right)(\log x)^{C_{3}}\right)
\end{aligned}
$$

or

$$
\begin{aligned}
\int_{0}^{t} Z^{\prime}(t-u) m_{f_{1}}\left(x^{u}\right) d u & =-\int_{0}^{t} \hat{Z}^{\prime}(t-u) m_{f}\left(x^{u}\right) d u+O\left(t^{C_{4}} H\left(x^{2 B_{1}}\right)(\log x)^{C_{3}}\right) \\
& =m_{f}\left(x^{t}\right)-m_{f_{1}}\left(x^{t}\right)+O\left(t^{C_{4}} H\left(x^{B_{1}}\right)(\log x)^{C_{3}}\right)
\end{aligned}
$$

which is (3.10) of Theorem 3.

Using Abel's summation on (3.10), we also derive the following estimate for functions satisfying (3.4) and (3.5) which we shall use in the next section to estimate $\Psi_{G}\left(x^{t}, x\right)$.

THEOREM 4. Let $f_{n}, n=1, \ldots, k$, be completely multiplicative functions satisfying (3.4) and (3.5). Then

$$
\begin{aligned}
& m_{f}\left(x^{t}\right)=m_{f_{1}}\left(x^{t}\right)+\int_{0}^{t} x^{2(t-u)} Z^{\prime}(t-u) m_{f_{1}}\left(x^{u}\right) d u
\end{aligned}
$$

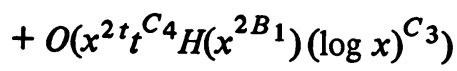

uniformly in $x$ and $t$ where $Z(t)$ satisfies (3.12), $m_{f_{1}}\left(x^{t}\right)$ is defined by (3.11), and $C_{3}$ and $C_{4}$ are absolute constants.

4. The estimate for $\Psi_{G}\left(x^{t}, x\right)$. We recall that we let $k=2, B_{1}=1$, $f_{1}(N \alpha)=1, f_{2}(N \alpha)=\epsilon(N \alpha)$, then

$$
m_{f}\left(x^{t}\right)=\Psi_{G}\left(x^{t}, x\right)
$$

and also that

$$
m_{f_{1}}\left(x^{t}\right)=\sum_{\alpha \in D\left(x^{t}\right)} 1
$$

and finally that

$$
L_{f_{1}}^{*}(x, y)=8 \log \min (x, y)+D+O\left(H\left(x^{2}\right)+H\left(y^{2}\right)\right)
$$

where $D$ is an absolute constant and $H(x)=O\left(\exp \left(-C(\log x)^{3 / 5-\delta}\right)\right)$ for $C>0$, $\delta>0$ from $\S 3$.

As a consequence of Theorem 4, we see that 


$$
\Psi_{G}\left(x^{t}, x\right)=m_{f_{1}}\left(x^{t}\right)+\int_{0}^{t} x^{2(t-u)} Z^{\prime}(t-u) m_{f_{1}}\left(x^{u}\right) d u
$$

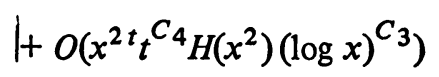

where $Z(t)$ satisfies (1.5) with $a=-1$.

Now we let

$$
m_{f_{1}}\left(x^{t}\right)=x^{2 t}\left\{\pi+R\left(x^{t}\right)\right\}
$$

From (1.8), we see that

$$
R\left(x^{t}\right)=o\left(x^{(b-2) t}\right)
$$

with $b<2 / 3$, an absolute constant.

Using (4.5) in (4.4), we prove the following estimate for $\Psi_{G}\left(x^{t}, x\right)$ which has (1.7) as a special case when $N=0$.

THEOREM 5. If $1 \leqslant t \leqslant(\log x)^{3 / 5-\delta}, \delta>0$ an arbitrary real number, $R(x)$ and $H(x)$ are defined by (4.5) and (3.8), respectively, then

$$
\begin{aligned}
\Psi_{G}\left(x^{t}, x\right)= & \pi x^{2 t}\left\{Z(t)+\sum_{m=0}^{N-1} \frac{(-1)^{m}}{m !} \frac{Z^{(m+1)}(t)}{\pi(\log x)^{m+1}} \int_{0}^{\infty} \frac{R(u)(\log u)^{m}}{u} d u\right\} \\
& +O_{N}\left(x^{2 t}\left\{t^{C_{4}} H\left(x^{2}\right)(\log x)^{C_{3}}+\frac{t\left|Z^{(N)}(t)\right|}{(\log x)^{N+1}}\right\}\right)
\end{aligned}
$$

for every natural number $N$. The estimate is uniform in $x$ and $t$ for $t$ outside the intervals $(n, n+\epsilon)$, where $n=1,2, \ldots, N+1$ are the discontinuities of $Z^{(N+1)}(t)$ and $\epsilon$ is an arbitrary positive real number.

Proof. After substituting (4.5) in (4.4), we have

$$
\Psi_{G}\left(x^{t}, x\right)=\pi x^{2 t}\left\{1+\int_{0}^{t} Z^{\prime}(t-u) d u+\pi^{-1} R\left(x^{t}\right)\right.
$$

$$
\left.+\pi^{-1} \int_{0}^{t} Z^{\prime}(t-u) R\left(x^{u}\right) d u+O\left(t^{C_{4}} H\left(x^{2}\right)(\log x)^{C_{3}}\right)\right\} .
$$

We note that $Z(t)=1+\int_{0}^{t} Z^{\prime}(t-u) d u$ and after the manner of Levin and Fainleib [3, Theorem 3.2.3], we let $\epsilon>0$ be so small that the interval $(t-\epsilon, t)$ does not contain any discontinuities of $Z^{(N+1)}(v), N$ is a natural number. Hence for $0<v<\epsilon$, we can apply Taylor's theorem to get

$$
Z^{\prime}(t-v)=\sum_{m=0}^{N-1} \frac{(-1)^{m}}{m !} Z^{(m+1)}(t-v) v^{m}+O\left(v^{N}\left|Z^{(N+1)}\left(t-\epsilon_{1}\right)\right|\right)
$$

for some $\epsilon_{1}$ such that $0<\epsilon_{1}<\epsilon$ (we note that the sum in (4.9) is empty if $N=0)$. 
Thus

$$
\begin{aligned}
\int_{0}^{\epsilon} Z^{\prime}(t-u) R\left(x^{u}\right) d u= & \sum_{m=0}^{N-1} \frac{(-1)^{m}}{m !} Z^{(m+1)}(t) \int_{0}^{\epsilon} u^{m} R\left(x^{u}\right) d u \\
& +o\left(\left|Z^{(N+1)}\left(t-\epsilon_{1}\right)\right| \int_{0}^{\epsilon} u^{N}\left|R\left(x^{u}\right)\right| d u\right) .
\end{aligned}
$$

The sum on the right-hand side of (4.10) is equal to

$$
\begin{aligned}
& \sum_{m=0}^{N-1} \frac{(-1)^{m}}{m !} \frac{Z^{(m+1)}(t)}{(\log x)^{m+1}} \int_{0}^{\infty} \frac{R(u)(\log u)^{m}}{u} d u \\
& -\sum_{m=0}^{N-1} \frac{(-1)^{m}}{m !} \frac{Z^{(m+1)}(t)}{(\log x)^{m+1}} \int_{x}^{\infty} \frac{R(u)(\log u)^{m}}{u} d u
\end{aligned}
$$

and the second sum of (4.11) is

$$
O_{N}\left(\frac{\left|Z^{\prime}(t)\right|}{\log x} \int_{x}^{\infty} \frac{|R(u)|}{u} d u\right)=O_{N}\left(\frac{x^{(b-2) \epsilon}\left|Z^{\prime}(t)\right|}{\log x}\right) .
$$

For the $O$-term of (4.10), we see that

$$
O\left(\left|Z^{(N+1)}\left(t-\epsilon_{1}\right)\right| \int_{0}^{\epsilon} u^{N}\left|R\left(x^{u}\right)\right| d u\right)=O_{N}\left(\frac{\left|Z^{(N+1)}\left(t-\epsilon_{1}\right)\right|}{(\log x)^{N+1}}\right) .
$$

Now $Z^{(N+1)}\left(t-\epsilon_{1}\right)=O\left(t\left|Z^{(N)}(t)\right|\right)$ and

$$
\begin{aligned}
\int_{\epsilon}^{t} Z^{\prime}(t-u) R\left(x^{u}\right) d u & =O\left(x^{(b-2) \epsilon} \int_{\epsilon}^{t}\left|Z^{\prime}(t-u)\right| d u\right) \\
& =O\left(x^{(b-2) \epsilon}(1+Z(t))\right) .
\end{aligned}
$$

Hence collecting these results in (4.8), we get (4.7) to complete the proof of Theorem 5 .

\section{BIBLIOGRAPHY}

1. J. H. Jordan, The divisibility of Gaussian integers by large Gaussian primes, Duke Math. J. 32 (1965), 503-509. MR 32 \#2392.

2. E. Landau, Vorlesungen über Zahlentheorie, Band 2, Leipzig, 1927, pp. 279-292.

3. B. V. Levin and A. S. Fainleib, Application of certain integral equations to questions of the theory of numbers, Uspehi Mat. Nauk 22 (1967), no. 3 (135), 119-197= Russian Math. Surveys 22 (1967), no. 3, 119-204. MR 37 \#5174.

4. K. Prachar, Primzahlverteilung, Springer-Verlag, Berlin, 1957. MR 19, 393.

DEPARTMENT OF MATHEMATICS, SOUTHWEST TEXAS STATE UNIVERSITY, SAN MARCOS, TEXAS 78666 